Research Article

\title{
Deep Learning-Based Correlation Analysis between Spine Surgery Lumbar Facet Joint and Lumbar Disc Herniation Using Magnetic Resonance Images
}

\author{
Chao Wang $(\mathbb{D}$, Jiabin Yuan $(\mathbb{D}$, Zebin Huang $\mathbb{D}$, and Zhicai Shi $\mathbb{D}$ \\ Department of Orthopedics, Changhai Hospital affiliated to Naval Medical University, Shanghai 200433, China \\ Correspondence should be addressed to Zhicai Shi; 201801010212@stu.hnie.edu.cn
}

Received 21 September 2021; Revised 9 November 2021; Accepted 11 November 2021; Published 6 December 2021

Academic Editor: M Pallikonda Rajasekaran

Copyright (c) 2021 Chao Wang et al. This is an open access article distributed under the Creative Commons Attribution License, which permits unrestricted use, distribution, and reproduction in any medium, provided the original work is properly cited.

\begin{abstract}
The research aimed at discussing the analytic function of convolutional neural network (CNN) algorithm-based magnetic resonance images (MRI) in the correlation between lumbar disc herniation (LDH) and angle and irregular variation of joint (IVJ) of lumbar facet-joint (LFJ). First, CNN-based MRI (CNNM) algorithm was constructed, and Markov random field (MRF) and fuzzy C-means (FCM) algorithms were introduced for comparison. Meanwhile, all patients received MRI examination of lumbar, and CNNM algorithm was adopted in MRI images. The results showed that the sensitivity, specificity, accuracy, and precision (98.53\%, 93.65\%, 99.56\%, and 98.74\%, respectively) of the CNNM algorithm were all superior to those of MRF algorithm (90.41\%, $81.11 \%, 91.18 \%$, and $91.13 \%$, respectively) and of FCM algorithm $(93.14 \%, 82.86 \%, 93.23 \%$, and 93.08\%, respectively) $(P<0.05)$. Besides, the lumbar spine angles of L3-L4, L4-L5, and L5-S1 $\left(6.03 \pm 1.34^{\circ}, 7.14 \pm 1.18^{\circ}\right.$, and $8.96 \pm 3.26^{\circ}$, respectively) in the experimental group was obviously less than those in the control group $\left(6.84 \pm 1.15^{\circ}, 9.85 \pm 1.25^{\circ}\right.$, and $17.34 \pm 4.79^{\circ}$, respectively) $(P<0.05)$. In the experimental group, there was irregular mutation of LFJ in 78 cases, while 8 cases suffered from irregular mutation of LFJ in the control group. The proportions of protrusion in L3/4, L4/5, and L5/S1 segments (11 cases, 53 cases, and 14 cases, respectively) was higher than that in the control group ( 1 case, 5 cases, and 2 cases, respectively) $(P<0.05)$. In short, the constructed CNNM algorithm had excellent performance in diagnosing lumbar MRI images and had clinical research and promotion value. Moreover, the IVJ of patients with LDH was notably increased, most of the physiological angle of the lumbar spine changed, and facet joint was correlated with the occurrence of LDH.
\end{abstract}

\section{Introduction}

Lumbar disc herniation (LDH) refers to the nucleus pulposus tissue protruding or protruding into the posterior or spinal canal due to the rupture of the lumbar intervertebral disc fibrous annulus, resulting in compression of adjacent nerve roots. It produces lumbar pain, pain, and numbness of the lower limbs, and even incontinence, paralysis of both lower limbs [1-3]. LDH is a spinal degenerative disease, which generally includes four types: bulging, protruding, free, and Schmorl snodes. It will cause radiating pain in lower limbs clinically, and it is also one of the most common diseases that affect the quality of life of patients in real life [4]. The causes of LDH include degeneration of the lumbar intervertebral disc, trauma, and genetics, among which lumbar intervertebral disc degeneration is the main factor. The intervertebral disc tissue bears the weight of the human trunk and upper limbs, and in daily life and work, the strain is heavier than other tissues [5]. As people age, the intervertebral discs will age. LFJ is the only movable joint of the spine, which has the functions of guiding the movement of the spine, resisting compression, shearing, and rotation. It bears $15 \%$ of the body's weight and has very complex biomechanical properties [6]. The direction of lumbar facetjoint (LFJ) is also an important factor affecting the overall movement and stability of the lumbar spine. Therefore, exploring the relationship between LFJ and LDH is also a hot topic in clinical efforts.

With the development of imaging, the current clinical examination methods for LDH include plain radiographs of 
the lumbar spine, computed tomography (CT) examinations, and magnetic resonance imaging (MRI) [7]. Lumbar $\mathrm{X}$-ray plain film is a traditional imaging detection method, which can better diagnose conventional lumbar disc disease and is cheap, but it is easily affected by objective factors, and misdiagnosis and missed diagnosis may occur $[8,9]$. CT examination can more clearly show the characteristics of lumbar degenerative disease, and it has the advantages of simple operation and reasonable price. However, the resolution of soft tissues is low, and there is no need to show the subtle damage of lumbar spine tissue in more detail. MRI is an imaging technology that adopts strong magnetic fields, magnetic field gradients, and radio waves, by which images of human organs are obtained. It has many advantages such as high resolution, multiple parameters, and high definition and has high sensitivity to measure the angle of lumbar facet joint [10]. In addition, MRI was highly sensitive to the lesions in human soft tissues, and it could effectively display facet joint lesions caused by early joint degeneration of patients. Furthermore, MRI caused no ionizing radiation injury, so this method was appropriate for examination on pregnant women, children, and other special population. Convolutional neural network $(\mathrm{CNN})$ is a kind of deep learning algorithms, which can learn to directly classify tasks from images, videos, text, or sound. Moreover, it works better in discovering patterns in images to identify objects, faces, and scenes. CNN has characterization learning capabilities, which learns directly from image data, and utilizes patterns to classify images without manual feature extraction $[11,12]$.

In summary, deep learning technology has a very eyecatching performance in the field of clinical imaging. The MRI recognition algorithm CNNM was constructed based on CNN, and the Markov random field (MRF) and fuzzy C-means (FCM) algorithms were introduced for comparison. Then, the CNNM algorithm was applied to the diagnosis of lumbar MRI images. By comparing the two groups of lumbar spines at L1-L2, L2-L3, L3-L4, L4-L5, and L5-S1 angle, as well as irregular variation of joint (IVJ) condition, the relationship between $\mathrm{LDH}$ and facet-joint angle and irregular changes was comprehensively explored.

\section{Materials and Methods}

2.1. Selection of Research Subjects. $102 \mathrm{LDH}$ patients who were admitted to the hospital from November 5, 2018, to June 20, 2020, were selected as the research subjects, and the age range was $18-65$ years. In addition, 58 normal volunteers who had their physical examination during the same period were taken as the control group. The study had been approved by the Medical Ethics Committee of Hospital, and the patients and their families understood the condition of the study and signed the informed consent forms.

Inclusion criteria: (1) patients older than 18 years; (2) patients with clinical symptoms of lower limb radiating pain; (3) patients who still could not relieve pain after conservative treatment; (4) patients who had clear consciousness and could cooperate with the examination normally; (5) patients who had no structural lumbar deformity in the past.
Exclusion criteria: (1) patients who had received lumbar spine surgery; (2) patients with other mental illnesses; (3) patients with lumbar scoliosis, lumbar spinal stenosis, or spinal fractures; (4) patients with poor MRI image quality that could not be diagnosed; (5) patients with incomplete clinical data; (6) patients with typical symptoms but negative imaging examination.

\subsection{MRI Scanning Method of Lumbar Intervertebral Disc.} The patients were examined with 3.0T magnetic resonance instrument. The patients were in a supine position and were fixed with an 8-channel knee coil. The scanning range was up to the lower endplate of the upper vertebral body and down to upper endplate of the lower vertebral body; for those protruding the nucleus pulposus upward or downward, the scanning area can be increased; and each intervertebral space was taken from top to bottom 3-4 cross sections. The scanning parameters were the matrix was $521 \times 521$, the layer thickness was $3 \mathrm{~mm}$, the layer spacing was $0.5 \mathrm{~mm}$, the field of view was $25 \times 25 \mathrm{~cm}$, the TR was $615 \mathrm{~ms}$, and the TE was $25 \mathrm{~ms}$. After the scan was completed, the image was sent for image reconstruction and diagnostic analysis, and then patient's lumbar facet-joint angle was measured.

Lumbar facet-joint angle measurement: the cross-sectional image of the intervertebral disc parallel to the endplate and close to the upper endplate of the lower vertebral body was selected, which was input into the computer processing software. Then, the image would be enlarged until the articular surface and articular process of LFJ were sufficiently clear. The angle between the joint line and the line connected by midpoint of the intervertebral disc and midpoint of the spinal process root was measured, to obtain the data of the joint angle. The sum of the joint angles on both sides was the joint angle value. The facet joint angle did not change according to the law of gradual increase. The form of the facet joint was the irregular change of the facet-joint angle, which included large-small-large (LSL) type, large-small-small (LSS) type, and small-large-small (SLS) type.

2.3. CNN-Based MRI Image Recognition Algorithm. There is a large amount of background information in the lumbar MRI image, which makes it difficult to recognize the lumbar disease manually. In addition, it may be affected by the patient's movement, so the resolution will be reduced. In Figure 1, it is more difficult to identify the lumbar spine due to patient movement, which increases the difficulty of medical examination and also increases the workload of doctors. Therefore, adopting artificial intelligence algorithms to automatically detect lumbar spine lesions becomes a hot research topic.

In this work, $\mathrm{CNN}$ is taken as a similarity function, then the twin CNN can be utilized for similarity learning. It is supposed that the MRI images of two different patients are $p$ and $q$, respectively, and then the similarity function compares the relationship between image $p$ and image $q$. The twin $\mathrm{CNN}$ is adopted to perform correlation conversion on the input image $p$ and image $q$, which is expressed as follows. 


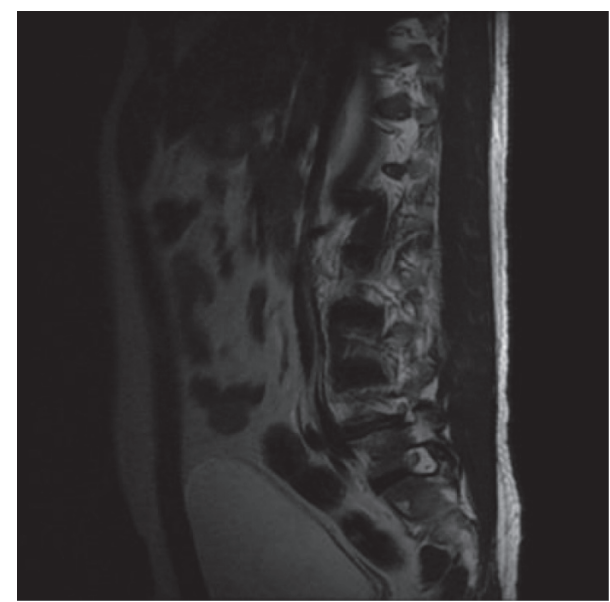

Figure 1: Schematic diagram of the lumbar spine.

$$
f(p, q)=d(\phi(p), \phi(q)) .
$$

Here, $d()$ is a function that represents a similarity measure, and $\phi$ is a convolution change coefficient. Given that the size of MRI candidate images of all patients is not completely the same, the fully convolutional network with respect to candidate images is adopted, which can bear the input of larger images and calculate the similarity of subwindows in the evaluation. The equation can be expressed as follows.

$$
f(p, q)=\phi(p) * \phi(q)+l 1
$$

Here, $l 1$ is a constant. The output of this equation is not a numerical value, but a score graph. In Figure 2, when the comparison image and the candidate image are input into the twin $\mathrm{CNN}$ at the same time, two output feature maps with different sizes can be obtained. The smaller feature map is taken as the convolution kernel to deconvolve the larger feature map, and finally, a score map will be obtained, and * represents the convolution operation. The position of the maximum value can be reversed back to the candidate image to find the most similar object position.

According to the maximum value of convolution score map compared to the position of the image center point and the total network step length, the target position of each search image is evaluated. Then, the cross-correlation combined feature map is adopted to independently evaluate the network of each subwindow. A single pair of positive and negative samples is taken as an example, and the training loss function of the network can be expressed as follows:

$$
h(m, n)=\log (1+\exp (-m n)) \text {. }
$$

In equation (3), $m$ represents the label value of the comparison sample and $m \in[-1,+1], n$ represents the true score of the candidate image calculated by the network, and $h(m, n)$ represents the training loss function. The loss function of the score map is defined as the mean of the loss of each sample, and it can be expressed as follows.

$$
H(m, n)=\frac{1}{|T|} \sum_{u \in T} h(m[u], n[u]) .
$$

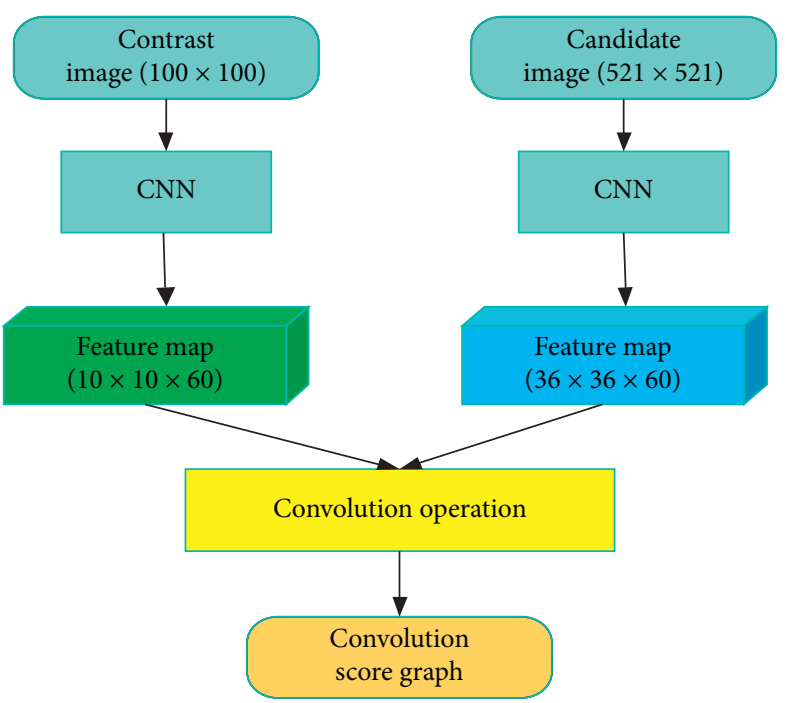

FIGURE 2: Schematic diagram of the smooth running of twin CNN.

In equation (4), $T$ represents the score map, $m[u]$ represents the position label in the score map, and $n[u]$ represents the comparison sample label. The distance between the element in the score map and the center are multiplied by $v$, and then, within a certain range, the element can be considered as a positive label, otherwise the element is considered as a negative sample. The available equation is as follows:

$$
m[u]= \begin{cases}-1, & \text { if } v\|u-b\| \leq r, \\ +1, & \text { otherwise. }\end{cases}
$$

In equation (5), $r$ represents the radius of the range, and $k$ represents a constant. Then, the stochastic gradient descent function is introduced to solve the loss function. Values similar to the convolution kernel usually get a higher response value, so inputting the image that is closest to the sample image can make the CNN learn more similar. Therefore, the search image centered on the above one target is input to the network, so as to avoid the output of all positions of the candidate image causing excessive computational complexity. The two CNN models are the same, which are expressed as follows.

$$
f(p, q)=f(q, p) .
$$

Given that the size of comparison image and search image are not the same, the normalization processing is conducted first. It is assumed that the length and width of the target frame are $a$ and $d$, respectively. The comparison image is $x^{2}$, the search image is $y^{2}$, and the two images are fixed to $x^{2}$; then, there is the following equation:

$$
G_{i}\left(a+2 k_{i}\right) \times G_{i}\left(d+2 k_{i}\right)=x^{2} .
$$

Here, $k_{i}$ represents the edge content, which is $1 / 2$ of the circumference of the target frame, and $k_{i}=a+d / 4$. Then, equation (7) can also be expressed as follows. 


$$
G_{i}=\frac{x}{\sqrt{(a+(a+d) / 2) \times(d+(a+d) / 2)}} .
$$

According to equation (8), a fixed-size contrast image can be obtained, and then more edge information is introduced to form a sample.

$$
\begin{aligned}
i & =\sqrt{(a+(a+d) / 2) \times(d+(a+d) / 2)}, \\
k_{x} & =\frac{y-x}{2 G_{i}}, \\
x & =i+2 k_{x} .
\end{aligned}
$$

In equations (9)-(11), $k_{x}$ represents edge information, and $x$ represents the size of the search image. Then, the final output image is enlarged 15 times by bicubic interpolation, and the distance vector between the position of the maximum value in the score map and the center can be expressed as follows.

$$
\overrightarrow{\mathrm{MC}}=(\alpha \max , \beta \max )-\frac{\left(\delta_{\text {center }}, \delta_{\text {center }}\right)}{2}
$$

Here, $(\alpha \max , \beta \max )$ represents the coordinates of the maximum value in the score map, and $\left(\delta_{\text {center }}, \delta_{\text {center }}\right)$ is the coordinates of center point of the score map. It is supposed that the total step length of convolution and pooling in the network is $s$, and then the distance vector between the center of the search image and current target position can be expressed as follows.

$$
\overrightarrow{\mathrm{CZ}}=C+\frac{\overrightarrow{\mathrm{MC}}}{16} \times s .
$$

Here, $C$ represents the center of the search image. The above equation is the lumbar MRI image recognition algorithm based on CNN, which is named as CNNM.

2.4. Simulation Experiment Design. The public lumbar spine database is utilized for training the fast and unsupervised detection algorithm of intervertebral disc based on MRF [13], and the lumbar detection algorithm FCM based on FCM clustering [14] is introduced to be compared with the CNNM algorithm constructed in this work. Sensitivity, specificity, accuracy, and precision are selected as indicators to evaluate the algorithm performance, which are calculated as follows.

$$
\begin{aligned}
\text { sensitivity } & =\frac{\mathrm{TP}}{\mathrm{TP}+\mathrm{FP}}, \\
\text { specificity } & =\frac{\mathrm{TN}}{\mathrm{TN}+\mathrm{FP}}, \\
\text { accuracy } & =\frac{\mathrm{TP}}{\mathrm{TP}+\mathrm{FN}}, \\
\text { precision } & =\frac{\mathrm{TP}+\mathrm{TN}}{\mathrm{TP}+\mathrm{TN}+\mathrm{FP}+\mathrm{FN}} .
\end{aligned}
$$

In equations (14)-(17), $\mathrm{TP}, \mathrm{TN}, \mathrm{FP}$, and $\mathrm{FN}$ mean true positive, true negative, false positive, and false negative, respectively.

2.5. Statistical Methods. Data processing was conducted via SPSS19.0 version statistical software analysis, mean\pm standard deviation $(\bar{x} \pm s)$ was how measurement data were expressed, and count data were in the form of percentage (\%). The sensitivity, specificity, accuracy, and precision of CNNM, MRF, and FCM algorithms were compared using paired $t$-test. The ratio of males and females, age, BMI, course of disease, lumbar spine L1-L2, L2-L3, L3-L4, L4-L5, and L5-S1 angles, and IVJ condition of two groups were compared by ANOVA. The difference was considerable at $P<0.05$.

\section{Results}

3.1. Contrast of Basic Information of Patients. Figures 3 and 4 presented the comparisons of the ratio of males and females, age, BMI, and course of disease. It was obvious that the male ratio, female ratio, age, $\mathrm{BMI}$, and course of disease in the experimental group were not obviously considerable in contrast to controls $(P>0.05)$.

Figure 5 revealed the MRI image of a male patient's lumbar spine. The sagittal plane showed the space-occupying lesions in the spinal canal at the L5 and S1 vertebral planes. The lesions showed slightly low signal on T1WI and mixed high signal on T2WI. The right edge extended into the right intervertebral foramen, which was not clearly demarcated from the nerve root, but did not protrude beyond the spinal canal.

\subsection{Algorithm Performance Evaluation Comparison.} Figure 6 and Figure 7 showed the comparison of sensitivity, specificity, accuracy, and precision of the three algorithms. The sensitivity, specificity, accuracy, and precision $(98.53 \%$, $93.65 \%, 99.56 \%$, and $98.74 \%$, respectively) of CNNM algorithm were obviously superior to those of MRF algorithm (90.41\%, 81.11\%, 91.18\%, and 91.13\%, respectively) and of FCM algorithm (93.14\%, 82.86\%, 93.23\%, and 93.08\%, respectively). The differences had statistical meaning $(P<0.05)$.

3.3. Contrast of Various Angles of Lumbar Spine between Groups. Figures 8 and 9 were comparisons of the angles of the lumbar spine at L1-L2, L2-L3, L3-L4, L4-L5, and L5-S1 between groups. The L1-L2 angle of the experimental group was $3.86 \pm 1.04^{\circ}$, the L2-L3 angle was $4.94 \pm 1.25^{\circ}$, the L3-L4 angle was $6.03 \pm 1.34^{\circ}$, the L4-L5 angle was $7.14 \pm 1.18^{\circ}$, and the L5-S1 angle was $8.96 \pm 3.26^{\circ}$. In the control group, the L1-L2 angle of the lumbar spine was $3.61 \pm 0.86^{\circ}$, the L2-L3 angle was $4.24 \pm 1.32^{\circ}$, the L3-L4 angle was $6.84 \pm 1.15^{\circ}$, the L4-L5 angle was $9.85 \pm 1.25^{\circ}$, and the L5-S1 angle was $17.34 \pm 4.79^{\circ}$. The L2-L3 angle of the lumbar spine in the experimental group was evidently larger in contrast to controls, with highly notable difference $(P<0.05)$. The 


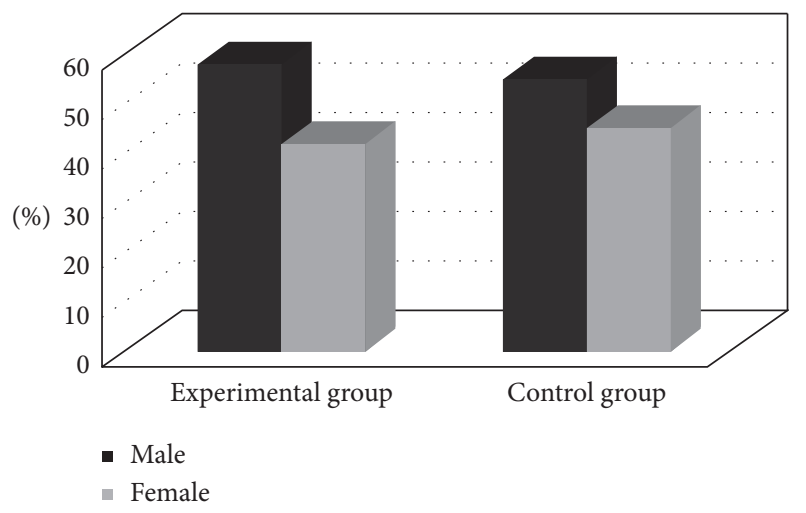

Figure 3: Comparison of the proportions of male and female between the two groups of patients.

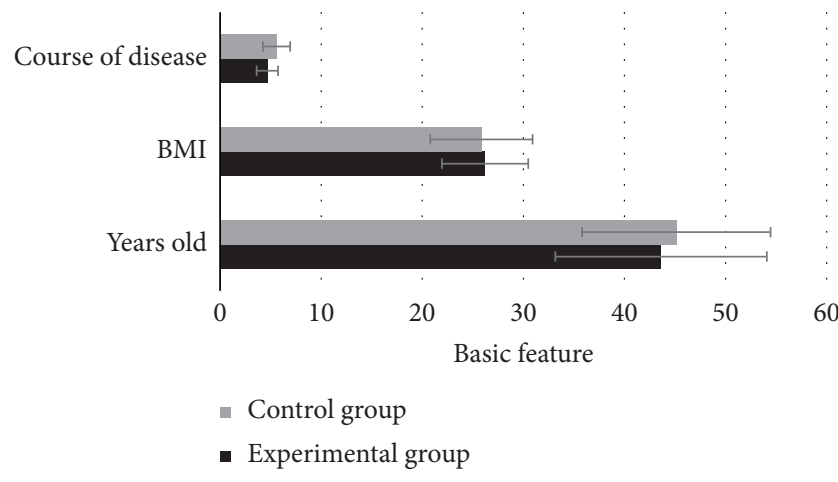

FIgURE 4: Contrast of age, BMI, and course of disease of patients.

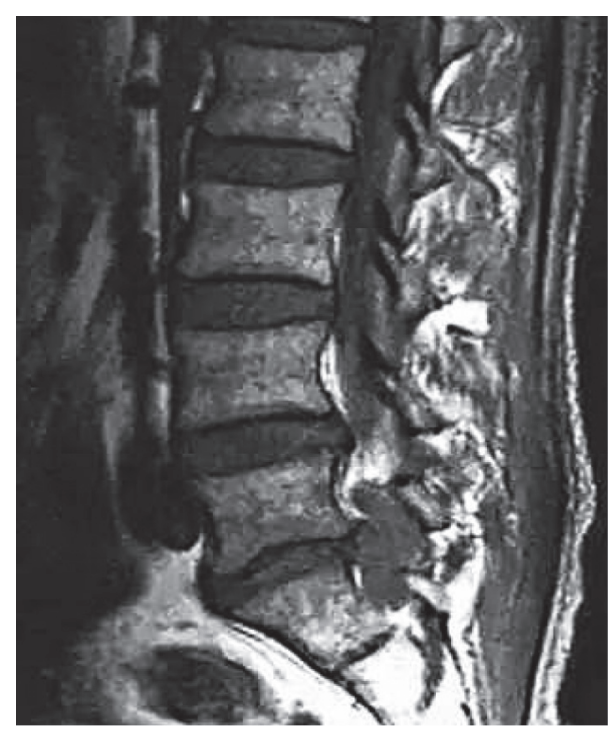

FIGURE 5: MRI image of lumbar spine in a male patient (aged 37 years old).

angles of L3-L4, L4-L5, and L5-S1 of the experimental group were obviously inferior to control group, and the difference was considerable $(P<0.05)$. Differences in the L1-L2 angles of the lumbar spine between groups were not substantial enough $(P>0.05)$.

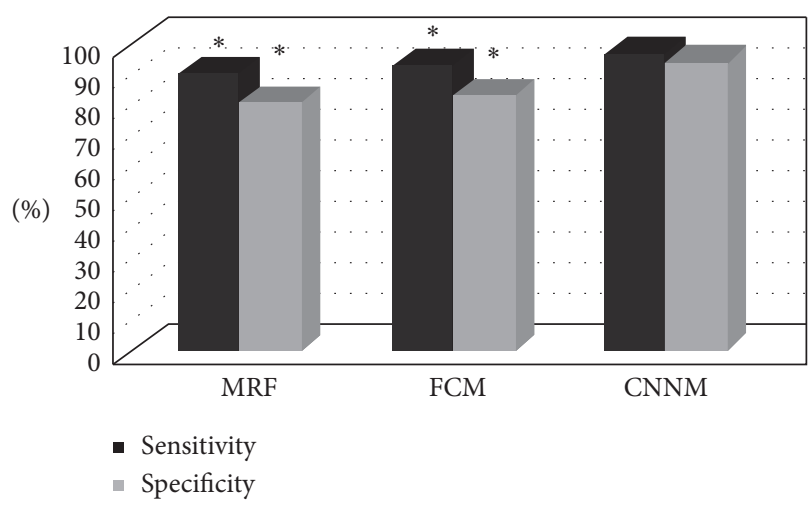

FIgURE 6: Contrast of recognition sensitivity and specificity of the three algorithms. Note: ${ }^{*}$ means that the difference was evident versus CNNM algorithm $(P<0.05)$.

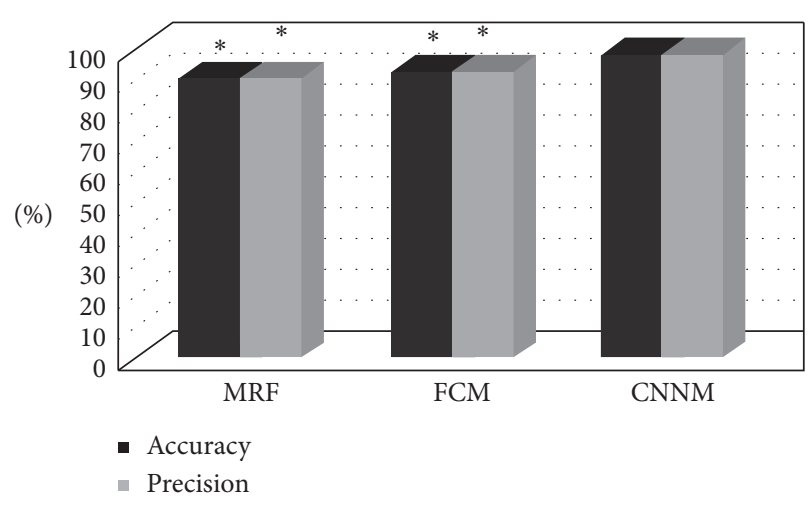

FIgURE 7: Contrast of recognition accuracy and precision of three algorithms. Note: ${ }^{*}$ means that the difference was evident versus CNNM algorithm $(P<0.05)$.

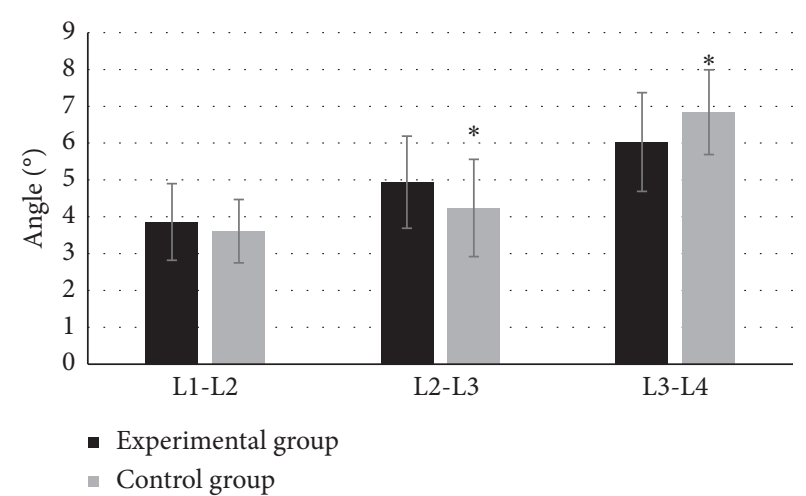

Figure 8: Contrast of L1-L2, L2-L3, and L3-L4 angles of the lumbar spine between groups. Note: ${ }^{*}$ means the difference was highly notable versus experimental group $(P<0.05)$, which means the same thing for all the figures.

3.4. Contrast of IVJ Conditions between Groups. Figure 10 shows the comparison of joint protrusion between the two groups. 78 patients in the experimental group had IVJ, and 8 patients in the control group had IVJ. In addition, in the experimental group, there were 11 cases with protrusion of L3/4, 53 cases with protrusion of L4/5, and 14 cases with protrusion of $\mathrm{L} 5 / \mathrm{S} 1$. In the control group, there was 1 case 


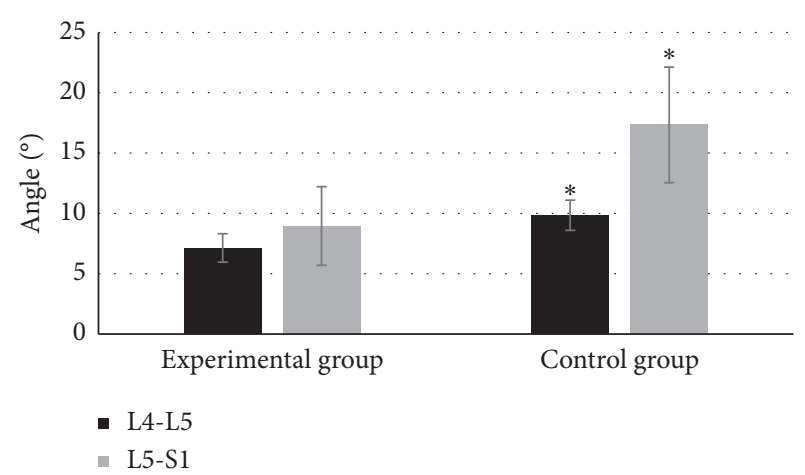

FIGURE 9: Contrast of L4-L5 and L5-S1 angles of the lumbar spine between groups.

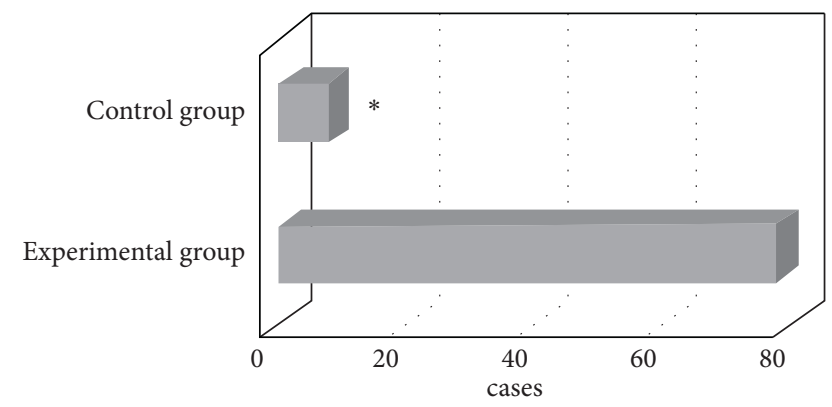

Figure 10: Comparison of the number of IVJ cases in the two groups.

with protrusion of $\mathrm{L} 3 / 4,5$ cases with protrusion of $\mathrm{L} 4 / 5$, and 2 cases with protrusion of L5/S1. The proportion of the number of protrusions in the L3/4, L4/5, and L5/S1 segments of experimental group was substantially higher relative to controls $(P<0.05)$, as shown in Figure 11.

Figure 12 shows the comparison of IVJ types between the two groups. Experimental group was with 34 cases of LSL type IVJ, 29 LSS type IVJ, and 15 SLS type IVJ. There were 2 cases of LSL type IVJ, 4 LSS type IVJ, and 2 SLS type IVJ in the control group. The number of LSL, LSS, and SLS type IVJ cases in the experimental group was higher versus the control group, with extremely evident difference $(P<0.05)$.

\section{Discussion}

The main symptom of LDH is low back and leg pain, which is a kind of degenerative diseases that affect people's quality of life. Clinical observations disclosed that the body's LFJ and the intervertebral disc shared the weight of the body. Moreover, the direction of the facet joint was an important factor affecting the mobility and stability of the lumbar spine, so there may be a close relationship between the facet joint and LDH $[15,16]$. First, the lumbar spine MRI image recognition algorithm CNNM was built based on CNN, and the fast and unsupervised detection algorithm MRF based on MRF and the lumbar detection algorithm FCM based on FCM clustering were introduced, which were compared with the proposed one regarding the algorithm performance. It was found that the recognition sensitivity, specificity,

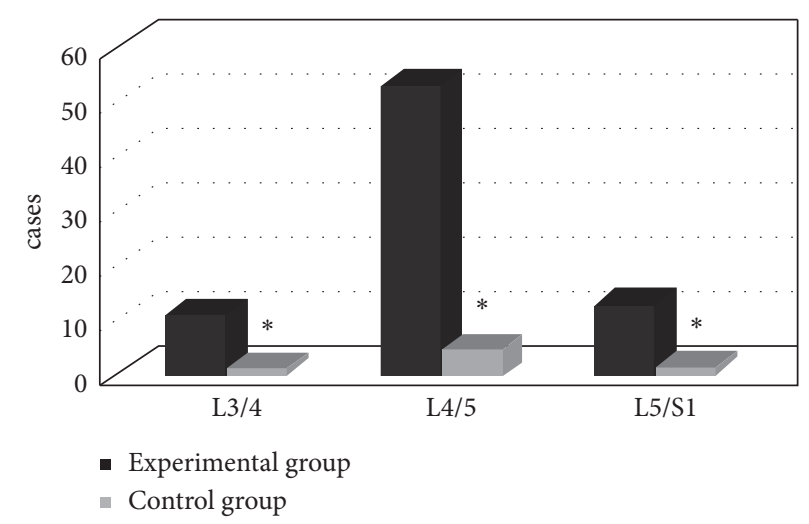

Figure 11: Comparison of joint protrusion between the two groups.

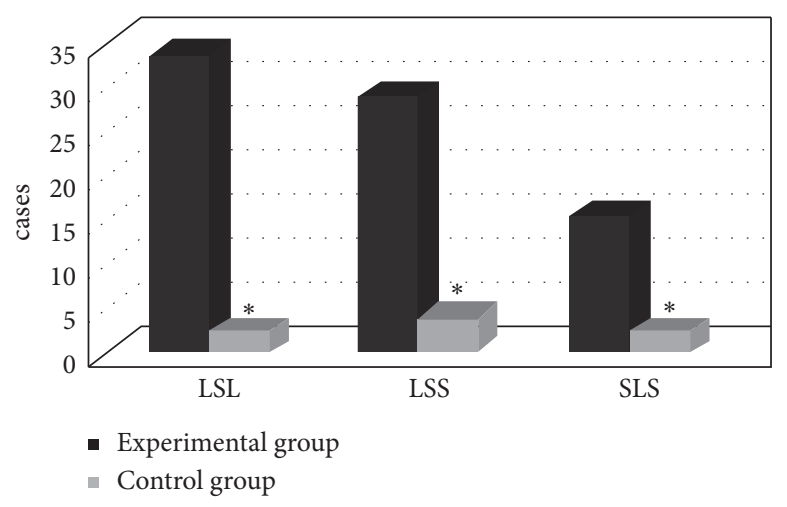

FIgURE 12: Contrast of IVJ types of patients.

accuracy, and precision of the CNNM algorithm were all greatly superior to MRF and FCM algorithms $(P<0.05)$. Such results were similar to the research results of Kuligowski et al. [17], indicating that the CNNM algorithm constructed in this work was superior to MRF and FCM algorithms in diagnosing lumbar MRI images, and had clinical research promotion value.

The L2-L3 angles of the experimental group were evidently larger than those of the control group, while the L3L4, L4-L5, and L5-S1 angles were not $(P<0.05)$. Li et al. [18] ever pointed out that the change of angles in the experimental group resulted from long-term lordosis of lumbar spines, thus paraspinal muscle showing high tension. Longstanding tension would cause lumbar strain, which even reflected to back or legs. Besides, spinal cord and nerve in human body were compressed by the physiological curvature of lumbar spine itself, which resulted in different types of neurological damage [19]. 78 patients in the experimental group had IVJ, and 8 patients in the control group had IVJ, which indicated that the IVJ of LDH patients increased notably. The proportion of the number of protrusions in the L3/4, L4/5, and L5/S1 segments of the experimental group was extremely higher versus the control group $(P<0.05)$, which was similar to the research results of Grubmüller et al. [20]. Segments L4/5 and L5/S1 were clinically prone to LDH lumbar segments. The results indicated that L4/5 and L5/S1 segments in patients with $\mathrm{LDH}$ 
was more likely to develop degenerative disease. The number of LSL, LSS, and SLS type IVJ cases in the experimental group was notably greater relative to the control group $(P<0.05)$, which indicated that the LFJ morphology type would cause different biomechanical lesions in the lumbar spine.

\section{Conclusion}

First, the lumbar spine MRI image recognition algorithm CNNM was constructed based on CNN, and MRF and FCM algorithms were introduced for comparison. Then, the CNNM algorithm was applied to lumbar spine MRI image diagnosis. It was found that the CNNM algorithm had excellent performance in diagnosing lumbar MRI images and had clinical research and promotion value. The IVJ of patients with LDH was considerably increased, most of the physiological angle of the lumbar spine changed, and facet joint was proved to be correlated with the occurrence of LDH. However, there are some shortcomings in this work. The sample size of patients is small, and only image maps are used for analysis. Later, it should consider increasing the sample size of patients and use histological, pathological, and biomechanical evidences to further analyze the relationship between LDH and facet joint. In short, the results provide a favorable theoretical basis for the clinical diagnosis and treatment of $\mathrm{LDH}$.

\section{Data Availability}

The data used to support the findings of this study are available from the corresponding author upon request.

\section{Conflicts of Interest}

The authors declare no conflicts of interest.

\section{References}

[1] E. Shokri, F. Kamali, E. Sinaei, and F. Ghafarinejad, "Spinal manipulation in the treatment of patients with MRI-confirmed lumbar disc herniation and sacroiliac joint hypomobility: a quasi-experimental study," Chiropractic \& Manual Therapies, vol. 26, no. 1, p. 16, 2018.

[2] D. S. Ziegler, L. Carreon, M. O. Andersen, and R. K. Jensen, "The association between preoperative MRI findings and surgical revision within three years after surgery for lumbar disc herniation," Spine, vol. 44, no. 11, pp. 818-825, 2019.

[3] J.-H. Kim, R. M. van Rijn, M. W. van Tulder et al., "Diagnostic accuracy of diagnostic imaging for lumbar disc herniation in adults with low back pain or sciatica is unknown; a systematic review," Chiropractic \& Manual Therapies, vol. 26, no. 1, p. 37, 2018.

[4] N. Thacker, S. Bakhshi, G. Chinnaswamy et al., "Management of non-hodgkin lymphoma: ICMR consensus document," Indian Journal of Pediatrics, vol. 84, no. 5, pp. 382-392, 2017.

[5] N. Karimi, P. Akbarov, and L. Rahnama, "Effects of segmental traction therapy on lumbar disc herniation in patients with acute low back pain measured by magnetic resonance imaging: a single arm clinical trial," Journal of Back and Musculoskeletal Rehabilitation, vol. 30, no. 2, pp. 247-253, 2017.
[6] N. B. Jain, J. Luz, L. D. Higgins et al., "The diagnostic accuracy of special tests for rotator cuff tear," American Journal of Physical Medicine \& Rehabilitation, vol. 96, no. 3, pp. 176-183, 2017.

[7] A. Mollo, A. Raffone, A. Travaglino et al., "Increased LDH5/ LDH1 ratio in preoperative diagnosis of uterine sarcoma with inconclusive MRI and LDH total activity but suggestive CT scan: a case report," BMC Women's Health, vol. 18, no. 1, p. 169, 2018.

[8] M. Annen, C. Peterson, S. Leemann, C. Schmid, B. Anklin, and B. K. Humphreys, "Comparison of outcomes in MRI confirmed lumbar disc herniation patients with and without modic changes treated with high velocity, low amplitude spinal manipulation," Journal of Manipulative and Physiological Therapeutics, vol. 39, no. 3, pp. 200-209, 2016.

[9] I. C. Benlidayi, S. Başaran, and G. Seydaoğlu, "Lumbosacral morphology in lumbar disc herniation: a chicken and egg issue," Acta Orthopaedica et Traumatologica Turcica, vol. 50, no. 3, pp. 346-350, 2016.

[10] K. Zheng, Z. Wen, and D. Li, "The clinical diagnostic value of lumbar intervertebral disc herniation based on MRI images," Journal of Healthcare Engineering, vol. 2021, Article ID 5594920, 9 pages, 2021.

[11] N. Oshima, R. Ishida, S. Kishimoto et al., "Dynamic imaging of $\mathrm{LDH}$ inhibition in tumors reveals rapid in vivo metabolic rewiring and vulnerability to combination therapy," Cell Reports, vol. 30, no. 6, pp. 1798-1810, 2020.

[12] P. Fritzell, C. Welinder-Olsson, B. Jönsson et al., "Bacteria: back pain, leg pain and Modic sign-a surgical multicentre comparative study," European Spine Journal, vol. 28, no. 12, pp. 2981-2989, 2019.

[13] S. Jafari, T. Dehesh, and F. Iranmanesh, "Classifying patients with lumbar disc herniation and exploring the most effective risk factors for this disease," Journal of Pain Research, vol. 12, pp. 1179-1187, 2019.

[14] O. Komolafe, S. P. Pereira, B. R. Davidson, and K. S. Gurusamy, "Serum C-reactive protein, procalcitonin, and lactate dehydrogenase for the diagnosis of pancreatic necrosis," Cochrane Database of Systematic Reviews, vol. 4, no. 4, Article ID CD012645, 2017.

[15] C. Zhang, L. Li, F. Y. Han et al., "Integrating fpmldhnactivated 19 F MRI agents for specific and sensitive detection of breast cancer," Small, vol. 15, no. 36, Article ID e1902309, 2019.

[16] D. H. Lee, P. Lee, S. W. Seo et al., "Neural substrates of cognitive reserve in Alzheimer's disease spectrum and normal aging," NeuroImage, vol. 186, pp. 690-702, 2019.

[17] T. Kuligowski, A. Dębiec-Bąk, and A. Skrzek, "Effectiveness of traction in young patients representing different stages of degenerative disc disease," Ortopedia Traumatologia Rehabilitacja, vol. 21, no. 3, pp. 187-196, 2019.

[18] J. Li, J. Jiang, Z. Chu et al., "Multiparametric MRI evaluation of liposomal prostaglandins E1 intervention on hepatic warm ischemia-reperfusion injury in rabbits," Journal of Magnetic Resonance Imaging, vol. 52, no. 1, pp. 217-228, 2020.

[19] S. J. Hong, D. Y. Kim, H. Kim, S. Kim, K. M. Shin, and S. S. Kang, "Resorption of massive lumbar disc herniation on MRI treated with epidural steroid injection: a retrospective study of 28 cases," Pain Physician, vol. 19, no. 6, pp. 381-388, 2016.

[20] B. Grubmüller, D. Senn, G. Kramer et al., "Response assessment using 68Ga-PSMA ligand PET in patients undergoing 177Lu-PSMA radioligand therapy for metastatic castration-resistant prostate cancer," European Journal of Nuclear Medicine and Molecular Imaging, vol. 46, no. 5, pp. 1063-1072, 2019. 